\title{
Mixed Convection Boundary Layer Flow over a Stretching Surface Filled with a Maxwell Fluid in Presence of Soret and Dufour Effects
}

\author{
Tasawar Hayat ${ }^{\mathrm{a}, \mathrm{b}}$, Meraj Mustafa ${ }^{\mathrm{a}}$, and Said Mesloub ${ }^{\mathrm{b}}$ \\ ${ }^{a}$ Department of Mathematics, Quaid-I-Azam University 45320, Islamabad 44000, Pakistan \\ a,b Department of Mathematics, College of Sciences, KS University, P. O. Box 2455, Riyadh 11451, Saudi Arabia \\ Reprint requests to T. H.; E-mail: pensy_t@yahoo.com
}

Z. Naturforsch. 65a, $401-410$ (2010); received June 26, 2009

\begin{abstract}
This article looks at the heat and mass transfer characteristics in mixed convection boundary layer flow about a linearly stretching vertical surface. An incompressible Maxwell fluid occupying the porous space takes into account the diffusion-thermo (Dufour) and thermal-diffusion (Soret) effects. The governing partial differential equations are transformed into a set of coupled ordinary differential equations, by invoking similarity transformations. The involved nonlinear differential system is solved analytically using the homotopy analysis method (HAM) to determine the convergent series expressions of velocity, temperature, and concentration. The physical interpretation to these expressions is assigned through graphs and tables for the Nusselt number $\theta^{\prime}(0)$ and the Sherwood number $\phi^{\prime}(0)$. The dependence of suction parameter $S$, mixed convection parameter $\lambda$, Lewis number $L e$, Prandtl number $\operatorname{Pr}$, Deborah number $\beta$, concentration buoyancy parameter $N$, porosity parameter $\gamma$, Dufour number $D f$, and Soret number $S r$ is seen on the flow quantities.
\end{abstract}

Key words: Series Solution; Maxwell Fluid; Heat Transfer.

\section{Introduction}

There have been several materials including polymer melts, drilling muds, oils and greases, clay coatings and suspensions, ketchup, cosmetic products, and many other which are non-Newtonian fluids. Confusion over the classification of non-Newtonian fluids stems because of the non-availability of a single model that displays all properties of such fluids. Inspite of this, the non-Newtonian fluids have been classified into three categories: (i) the differential type, (ii) the rate type, and (iii) the integral type. Note that as a consequence of diversity in the physical structures the non-Newtonian fluids do not have a single constitutive equation. Thus several models of such fluids have been proposed and most of them being empirical or semi-empirical. The method of continuum mechanics is required for general three-dimensional representation. Many key questions are thus unresolved. Some of the proposed models do not agree with experiments. Hence in practical applications, empirical or semiempirical equations have been invoked. A host of recent researchers [1-10] have looked at the flows of non-Newtonian fluids in different situations.

There is a simplest subclass of rate type fluids which is known as the Maxwell fluid. The aim of this investigation is to discuss the mixed convection flow of a
Maxwell fluid occupying a porous medium. The fluid is bounded by a porous stretching surface. The boundary layer flow is examined with Soret and Dufour effects. The homotopy analysis method [11-25] is used in the computations of series solutions. The role of pertinent parameters in the series solution is discussed by plotting graphs.

\section{Development of Problems}

We examine heat and mass transfer flow due to stretching of a heated or cooled vertical surface of variable temperature $T_{\mathrm{w}}(x)$ and variable concentration $C_{\mathrm{w}}(x)$ in a porous medium filled with a Maxwell fluid of uniform ambient temperature $T_{\infty}$ and uniform ambient concentration $C_{\infty}$. The surface is stretched in its plane with the velocity $u_{\mathrm{w}}(x)$. The density variation and the buoyancy effects are taken into account and the Boussinesq approximation for both the temperature and concentration gradient is adopted. The Soret and the Dufour effects are also retained. The steady boundary layer equations in absence of heat generation and viscous dissipation are given by the following equations:

$$
\frac{\partial u}{\partial x}+\frac{\partial v}{\partial y}=0
$$




$$
\begin{aligned}
& u \frac{\partial u}{\partial x}+v \frac{\partial u}{\partial y}=v \frac{\partial^{2} u}{\partial y^{2}} \\
& -\lambda_{1}\left(u^{2} \frac{\partial^{2} u}{\partial x^{2}}+v^{2} \frac{\partial^{2} u}{\partial y^{2}}+2 u v \frac{\partial^{2} u}{\partial x \partial y}\right) \\
& -\frac{v}{K} u+g\left[\beta_{\mathrm{T}}\left(T-T_{\infty}\right)+\beta_{\mathrm{C}}\left(C-C_{\infty}\right)\right], \\
& u \frac{\partial T}{\partial x}+v \frac{\partial T}{\partial y}=\alpha_{m} \frac{\partial^{2} T}{\partial y^{2}}+\frac{D_{\mathrm{e}} k_{\mathrm{T}}}{C_{\mathrm{s}} C_{\mathrm{p}}} \frac{\partial^{2} C}{\partial y^{2}}, \\
& u \frac{\partial C}{\partial x}+v \frac{\partial C}{\partial y}=D_{\mathrm{e}} \frac{\partial^{2} C}{\partial y^{2}}+\frac{D_{\mathrm{e}} k_{\mathrm{T}}}{T_{m}} \frac{\partial^{2} T}{\partial y^{2}} .
\end{aligned}
$$

The appropriate boundary conditions are

$$
\begin{aligned}
& u=U_{\mathrm{w}}(x)=a x, \quad v=-V_{0}, \\
& T=T_{\mathrm{w}}(x)=T_{\infty}+b x, \quad C=C_{\mathrm{w}}(x)=C_{\infty}+c x \\
& \text { at } y=0, \\
& u \rightarrow 0, \quad \frac{\partial u}{\partial y} \rightarrow 0, \quad T \rightarrow T_{\infty}, \quad C \rightarrow C_{\infty} \\
& \text { as } y \rightarrow \infty .
\end{aligned}
$$

In the statement of problems, $x$ and $y$ are the Cartesian coordinates along and normal to the plate, respectively, $u$ and $v$ are the velocity components along $x$ and $y$-axes, $T$ is the fluid temperature, $g$ is the gravitational acceleration, $\alpha$ is the thermal diffusivity, $\beta_{\mathrm{T}}$ is the coefficient of thermal expansion, and $\lambda_{1}$ is a relaxation time. Further $a(>0), b$, and $c(>0)$ are constants with $b>0$ for a heated plate $\left(T_{\mathrm{w}}>T_{\infty}\right)$ and $b<0$ for a cooled surface $\left(T_{\mathrm{W}}<T_{\infty}\right)$, respectively.

Defining

$$
\begin{aligned}
& \psi=x \sqrt{a v} f(\eta), \quad \theta(\eta)=\frac{T-T_{\infty}}{T_{\mathrm{w}}-T_{\infty}}, \\
& \phi(\eta)=\frac{C-C_{\infty}}{C_{\mathrm{w}}-C_{\infty}}, \quad \eta=\sqrt{\frac{a}{v}} y,
\end{aligned}
$$

and invoking (2) - (4), we have

$$
\begin{aligned}
& f^{\prime \prime \prime}+f f^{\prime \prime}-f^{\prime 2}+\beta\left(2 f f^{\prime} f^{\prime \prime}-f^{2} f^{\prime \prime \prime}\right) \\
& -\gamma f^{\prime}+\lambda(\theta+N \phi)=0, \\
& \frac{1}{P r} \theta^{\prime \prime}+f \theta^{\prime}-\theta f^{\prime}+D f \phi^{\prime \prime}=0, \\
& \phi^{\prime \prime}+\operatorname{Pr} L e\left(f \phi^{\prime}-\phi f^{\prime}\right)+S r L e \theta^{\prime \prime}=0, \\
& f(0)=S, \quad f^{\prime}(0)=1, \quad \theta(0)=1, \\
& \phi(0)=1, \quad f^{\prime}(\infty) \rightarrow 0, \quad f^{\prime \prime}(\infty) \rightarrow 0, \\
& \theta(\infty) \rightarrow 0, \quad \phi(\infty) \rightarrow 0,
\end{aligned}
$$

T. Hayat et al. · Stretching Surface Filled with a Maxwell Fluid

where $\operatorname{Pr}=v / \alpha_{m}$ is the Prandtl number, $L e=$ $\alpha_{m} / D e$ is the Lewis number, $\lambda$ is the dimensionless mixed convection parameter, $\beta(\geq 0)$ is the Deborah number, $N$ is the constant dimensionless concentration buoyancy parameter, $\gamma$ is the dimensionless porosity parameter, $D f$ is the Dufour number, and $S r$ is the Soret number. These are given by

$$
\begin{aligned}
& \lambda=\frac{g \beta_{\mathrm{T}} b}{a^{2}}=\frac{g \beta_{\mathrm{T}}\left(T_{\mathrm{w}}-T_{\infty}\right) x^{3} / v^{2}}{u_{\mathrm{w}}^{2} x^{2} / v^{2}}=\frac{G r_{x}}{R e_{x}^{2}}, \\
& \beta=\lambda a, \quad \gamma=\frac{v}{a K}, \quad N=\frac{\beta_{\mathrm{C}}}{\beta_{\mathrm{T}}} \frac{\left(C_{\mathrm{w}}-C_{\infty}\right)}{\left(T_{\mathrm{w}}-T_{\infty}\right)}, \\
& D f=\frac{D_{\mathrm{e}} k_{\mathrm{T}}}{C_{\mathrm{s}} C_{\mathrm{p}}} \frac{\left(C_{\mathrm{w}}-C_{\infty}\right)}{\left(T_{\mathrm{w}}-T_{\infty}\right) v}, \quad S r=\frac{D_{\mathrm{e}} k_{\mathrm{T}}}{T_{m} \alpha_{m}} \frac{\left(T_{\mathrm{w}}-T_{\infty}\right)}{\left(C_{\mathrm{w}}-C_{\infty}\right)} .
\end{aligned}
$$

with $G r_{x}=g \beta_{\mathrm{T}}\left(T_{\mathrm{w}}-T_{\infty}\right) x^{3} / v^{2}$ being the local Grashof number and $\operatorname{Re}_{x}=u_{\mathrm{w}} x / v$ is the local Reynolds number. Note that $\lambda>0$ holds for assisting flow (heated plate), $\lambda<0$ corresponds to opposing flow (cooled plate), and $\lambda=0$ for forced convection flow. Furthermore, $N$ may have positive values $(N>0)$, negative values $(N<0)$, and $N=0$ (when mass transfer is absent).

The local Nusselt number $N u_{x}$ and the local Sherwood number $S h$ are given by

$$
N u_{x}=\frac{x q_{\mathrm{w}}}{k\left(T_{\mathrm{w}}-T_{\infty}\right)}, \quad S h=\frac{x j_{\mathrm{w}}}{D e\left(T_{\mathrm{w}}-T_{\infty}\right)},
$$

where $\tau_{\mathrm{w}}, q_{\mathrm{w}}$, and $j_{\mathrm{w}}$ are the wall skin friction, the wall heat flux, and the mass flux from the plate, which are defined as

$$
q_{\mathrm{w}}=-k\left(\frac{\partial T}{\partial y}\right)_{y=0}, \quad j_{\mathrm{w}}=-D e\left(\frac{\partial C}{\partial y}\right)_{y=0} .
$$

In terms of dimensionless quantites one has

$$
N u_{x} / R e_{x}^{1 / 2}=-\theta^{\prime}(0), \quad S h / R e_{x}^{1 / 2}=-\phi^{\prime}(0) .
$$

\section{Homotopy Analysis Solutions}

The velocity $f(\eta)$, the temperature $\theta(\eta)$, and the concentration field $\phi(\eta)$ in terms of the set of base functions

$$
\left\{\eta^{k} \exp (-n \eta) \mid k \geq 0, n \geq 0\right\}
$$

can be expressed as

$$
f(\eta)=a_{0,0}^{0}+\sum_{n=0}^{\infty} \sum_{k=0}^{\infty} a_{m, n}^{k} \eta^{k} \exp (-n \eta),
$$




$$
\begin{aligned}
& \theta(\eta)=\sum_{n=0}^{\infty} \sum_{k=0}^{\infty} b_{m, n}^{k} \eta^{k} \exp (-n \eta), \\
& \phi(\eta)=\sum_{n=0}^{\infty} \sum_{k=0}^{\infty} c_{m, n}^{k} \eta^{k} \exp (-n \eta),
\end{aligned}
$$

in which $a_{m, n}^{k}, b_{m, n}^{k}$, and $c_{m, n}^{k}$ are the coefficients. By rule of solution expressions and the boundary conditions (10), one can choose the initial guesses $f_{0}, \theta_{0}$, and $\phi_{0}$ of $f(\eta), \theta(\eta)$, and $\phi(\eta)$ as

$$
\begin{aligned}
& f_{0}(\eta)=S+1-\exp (-\eta), \\
& \theta_{0}(\eta)=\exp (-\eta) \\
& \phi_{0}(\eta)=\exp (-\eta)
\end{aligned}
$$

The auxiliary linear operators are

$$
\begin{aligned}
& \mathcal{L}_{f}=\frac{\mathrm{d}^{3} f}{\mathrm{~d} \eta^{3}}-\frac{\mathrm{d} f}{\mathrm{~d} \eta}, \\
& \mathcal{L}_{\theta}=\frac{\mathrm{d}^{2} \theta}{\mathrm{d} \eta^{2}}-\theta, \\
& \mathcal{L}_{\phi}=\frac{\mathrm{d}^{2} \phi}{\mathrm{d} \eta^{2}}-\phi .
\end{aligned}
$$

Note that the above operators possess the following properties:

$$
\begin{aligned}
& \mathcal{L}_{f}\left[C_{1}+C_{2} \exp (\eta)+C_{3} \exp (-\eta)\right]=0, \\
& \mathcal{L}_{\theta}\left[C_{4} \exp (\eta)+C_{5} \exp (-\eta)\right]=0, \\
& \mathcal{L}_{\phi}\left[C_{6} \exp (\eta)+C_{7} \exp (-\eta)\right]=0,
\end{aligned}
$$

where $c_{i}(i=1-7)$ are the arbitrary constants.

If $p \in[0,1]$ is an embedding parameter and $\hbar_{f}, \hbar_{\theta}$, and $\hbar_{\phi}$ denote the non-zero auxiliary parameters, respectively, then the zeroth-order deformation problems are constructed as follows:

$$
\begin{aligned}
& (1-p) \mathcal{L}_{f}\left[\hat{f}(\eta, p)-f_{0}(\eta)\right]= \\
& p \hbar_{f} \mathcal{N}_{f}[\hat{f}(\eta, p), \hat{\theta}(\eta, p), \hat{\phi}(\eta, p)], \\
& (1-p) \mathcal{L}_{\theta}\left[\hat{\theta}(\eta, p)-\theta_{0}(\eta)\right]= \\
& p \hbar_{\theta} \mathcal{N}_{\theta}[\hat{f}(\eta, p), \hat{\theta}(\eta, p), \hat{\phi}(\eta, p)], \\
& (1-p) \mathcal{L}_{\phi}\left[\hat{\phi}(\eta, p)-\phi_{0}(\eta)\right]= \\
& p \hbar_{\phi} \mathcal{N}_{\phi}[\hat{f}(\eta, p), \hat{\theta}(\eta, p), \hat{\phi}(\eta, p)], \\
& \left.\hat{f}(\eta ; p)\right|_{\eta=0}=S,\left.\quad \frac{\partial \hat{f}(\eta ; p)}{\partial \eta}\right|_{\eta=0}=1, \\
& \left.\frac{\partial \hat{f}(\eta ; p)}{\partial \eta}\right|_{\eta=\infty}=0,
\end{aligned}
$$

$$
\begin{aligned}
& \left.\hat{\theta}(\eta ; p)\right|_{\eta=0}=1,\left.\quad \hat{\theta}(\eta ; p)\right|_{\eta=\infty}=0, \\
& \left.\hat{\phi}(\eta ; p)\right|_{\eta=0}=1,\left.\quad \hat{\phi}(\eta ; p)\right|_{\eta=\infty}=0,
\end{aligned}
$$

in which the nonlinear operators $\mathcal{N}_{f}, \mathcal{N}_{\theta}$, and $\mathcal{N}_{\phi}$ are

$$
\begin{aligned}
& \mathcal{N}_{f}[\hat{f}(\eta ; p), \hat{\theta}(\eta ; p), \hat{\phi}(\eta ; p)]= \\
& \quad \frac{\partial^{3} \hat{f}(\eta, p)}{\partial \eta^{3}}+\hat{f}(\eta, p) \frac{\partial^{2} \hat{f}(\eta, p)}{\partial \eta^{2}} \\
& \quad-\left(\frac{\partial \hat{f}(\eta, p)}{\partial \eta}\right)^{2}-\gamma \frac{\partial \hat{f}(\eta, p)}{\partial \eta} \\
& +\beta\left\{2 \hat{f}(\eta, p) \frac{\partial \hat{f}(\eta, p)}{\partial \eta} \frac{\partial^{2} \hat{f}(\eta, p)}{\partial \eta^{2}}\right. \\
& \left.\quad-(\hat{f}(\eta, p))^{2} \frac{\partial^{3} \hat{f}(\eta, p)}{\partial \eta^{3}}\right\} \\
& +\lambda(\hat{\theta}(\eta, p)+N \hat{\phi}(\eta, p)),
\end{aligned}
$$

$$
\left.-\frac{\partial \hat{f}(\eta ; p)}{\partial \eta} \hat{\phi}(\eta ; p)\right)+\operatorname{SrLe} \frac{\partial^{2} \hat{\theta}(\eta, p)}{\partial \eta^{2}}
$$

When $p=0$ and $p=1$, we have

$$
\begin{aligned}
& \hat{f}(\eta ; 0)=f_{0}(\eta), \quad \hat{f}(\eta ; 1)=f(\eta), \\
& \hat{\theta}(\eta ; 0)=\theta_{0}(\eta), \quad \hat{\theta}(\eta ; 1)=\theta(\eta), \\
& \hat{\phi}(\eta ; 0)=\phi_{0}(\eta), \quad \hat{\phi}(\eta ; 1)=\phi(\eta) .
\end{aligned}
$$

By Taylor's theorem

$$
\begin{aligned}
& \hat{f}(\eta ; p)=f_{0}(\eta)+\sum_{m=1}^{\infty} f_{m}(\eta) p^{m}, \\
& \hat{\theta}(\eta ; p)=\theta_{0}(\eta)+\sum_{m=1}^{\infty} \theta_{m}(\eta) p^{m} \\
& \hat{\phi}(\eta ; p)=\phi_{0}(\eta)+\sum_{m=1}^{\infty} \phi_{m}(\eta) p^{m}
\end{aligned}
$$


404

$$
\begin{aligned}
& f_{m}(\eta)=\left.\frac{1}{m !} \frac{\partial^{m} f(\eta ; p)}{\partial \eta^{m}}\right|_{p=0}, \\
& \theta_{m}(\eta)=\left.\frac{1}{m !} \frac{\partial^{m} \theta(\eta ; p)}{\partial \eta^{m}}\right|_{p=0}, \\
& \phi_{m}(\eta)=\left.\frac{1}{m !} \frac{\partial^{m} \phi(\eta ; p)}{\partial \eta^{m}}\right|_{p=0} .
\end{aligned}
$$

The auxiliary parameters are so properly chosen that the series (39)-(41) converge at $p=1$, then we have

$$
\begin{aligned}
& f(\eta)=f_{0}(\eta)+\sum_{m=1}^{\infty} f_{m}(\eta), \\
& \theta(\eta)=\theta_{0}(\eta)+\sum_{m=1}^{\infty} \theta_{m}(\eta), \\
& \phi(\eta)=\phi_{0}(\eta)+\sum_{m=1}^{\infty} \phi_{m}(\eta) .
\end{aligned}
$$

The $m$ th-order deformation problems are of the form

$$
\begin{gathered}
\mathcal{L}_{f}\left[f_{m}(\eta)-\chi_{m} f_{m-1}(\eta)\right]=\hbar_{f} \mathcal{R}_{m}^{f}(\eta) \\
\mathcal{L}_{\theta}\left[\theta_{m}(\eta)-\chi_{m} \theta_{m-1}(\eta)\right]=\hbar_{\theta} \mathcal{R}_{m}^{\theta}(\eta) \\
\mathcal{L}_{\phi}\left[\phi_{m}(\eta)-\chi_{m} \phi_{m-1}(\eta)\right]=\hbar_{\phi} \mathcal{R}_{m}^{\phi}(\eta) \\
f_{m}(0)=0, \quad f_{m}^{\prime}(0)=0, \quad f_{m}^{\prime}(\infty)=0 \\
\theta_{m}(0)=0, \quad \theta_{m}(\infty)=0, \\
\text { and } \phi_{m}(0)=0, \quad \phi_{m}(\infty)=0 \\
\mathcal{R}_{m}^{f}(\eta)=f_{m-1}^{\prime \prime \prime}-\gamma f_{m-1}^{\prime}+\sum_{k=0}^{m-1}\left[f_{m-1-k} f_{k}^{\prime \prime}\right. \\
-f_{m-1-k}^{\prime} f_{k}^{\prime}+\beta\left\{2 f_{m-1-k} \sum_{l=0}^{\prime} f_{k-l}^{\prime} f_{l}^{\prime \prime}\right. \\
\left.\left.-f_{m-1-k} \sum_{l=0}^{k} f_{k-l} f_{l}^{\prime \prime \prime}\right\}+\lambda\left(\theta_{m-1-k}+N \phi_{m-1-k}\right)\right] \\
\mathcal{R}_{m}^{\theta}(\eta)=\frac{1}{P r} \theta_{m-1}^{\prime \prime} \\
\chi_{m}=\left\{\begin{array}{l}
\mathcal{R}_{m}^{\phi}(\eta)=\phi_{m-1}^{m-1} \\
+\sum_{k=0}^{\prime \prime}\left[f_{m-1-k} \theta_{k}^{\prime}-\theta_{m-1-k} f_{k}^{\prime}\right]+D f \phi_{m-1}^{\prime \prime} \\
+\operatorname{Pr}_{2} \sum_{k=0}^{m-1}\left[f_{m-1-k} \phi_{k}^{\prime}-\phi_{m-1-k} f_{k}^{\prime}\right]+S r L e \theta_{m-1}^{\prime \prime}
\end{array}\right.
\end{gathered}
$$

T. Hayat et al. · Stretching Surface Filled with a Maxwell Fluid

Table 1. Convergence of HAM solutions for different order of approximations when $S=0.5, \beta=0.1, \gamma=\lambda=P r=L e=$ $1.0, D f=0.1$, and $S r=0.2$.

\begin{tabular}{cccc}
\hline Order of approximations & $-f^{\prime \prime}(0)$ & $-\theta^{\prime}(0)$ & $-\phi^{\prime}(0)$ \\
\hline 1 & 1.277000 & 1.167000 & 1.127000 \\
5 & 1.319417 & 1.200177 & 1.103297 \\
10 & 1.319487 & 1.200257 & 1.103187 \\
15 & 1.319487 & 1.200227 & 1.103217 \\
20 & 1.319487 & 1.200227 & 1.103217 \\
25 & 1.319487 & 1.200227 & 1.103217 \\
30 & 1.319487 & 1.200227 & 1.103217 \\
35 & 1.319487 & 1.200227 & 1.103217 \\
40 & 1.319487 & 1.200227 & 1.103217 \\
50 & 1.319487 & 1.200227 & 1.103217 \\
\hline
\end{tabular}

and the general solutions of (46) - (48) are

$$
\begin{array}{r}
f_{m}(\eta)=f_{m}^{*}(\eta)+C_{1}+C_{2} \exp (\eta)+C_{3} \exp (-\eta) \\
\theta_{m}(\eta)=\theta_{m}^{*}(\eta)+C_{4} \exp (\eta)+C_{5} \exp (-\eta),
\end{array}
$$

where $f_{m}^{*}(\eta), \theta_{m}^{*}(\eta)$, and $\phi_{m}^{*}(\eta)$ denote the special solutions and

$$
\begin{aligned}
& C_{2}=C_{4}=C_{6}=0, \quad C_{1}=-C_{3}-f_{m}^{*}(0), \\
& C_{3}=\left.\frac{\partial f^{*}(\eta)}{\partial \eta}\right|_{\eta=0}, \quad C_{5}=-\theta_{m}^{*}(0), \\
& C_{7}=-\phi_{m}^{*}(0) .
\end{aligned}
$$

\section{Convergence of the Homotopy Solutions}

We note that the solutions (46) - (48) consist of $\hbar_{f}$, $\hbar_{\theta}$, and $\hbar_{\phi}$ which can adjust and control the convergence of the series solutions. In order to find the admissible values of $\hbar_{f}, \hbar_{\theta}$, and $\hbar_{\phi}$ of the functions $f^{\prime \prime}(0)$, $\theta^{\prime}(0)$, and $\phi^{\prime}(0)$ the $\hbar_{f}, \hbar_{\theta}$, and $\hbar_{\phi}$-curves are displayed for the 15th-order of approximations. It is obvious from Figure 1 that the range for the admissible

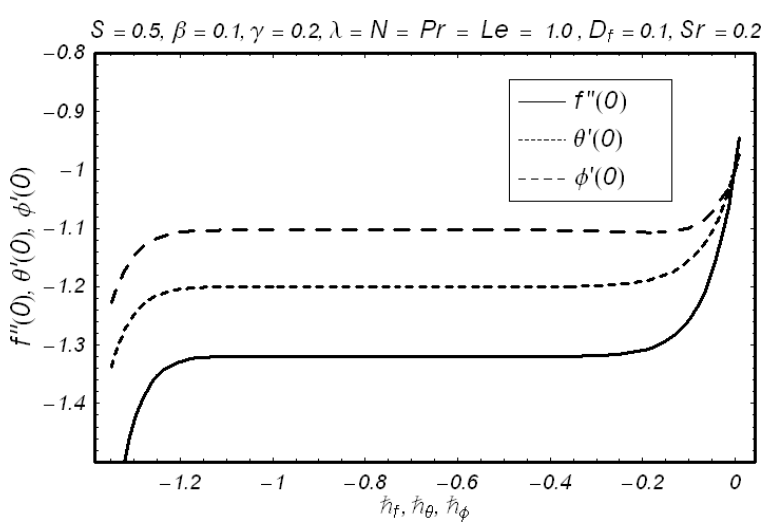

Fig. 1. $\hbar$-curve for the functions $f, \theta$, and $\phi$. 
Table 2. Values of the local Nusselt number $R e_{x}^{-1 / 2} N u_{x}$ and the local Sherwood number $R e_{x}^{-1 / 2} S h$ for some values of $S$, $\beta, \gamma, \lambda$, and $N$ when $\operatorname{Pr}=L e=1.0, D f=0.1, S r=0.2$.

\begin{tabular}{ccccccc}
\hline$S$ & $\beta$ & $\gamma$ & $\lambda$ & $N$ & $-\theta^{\prime}(0)$ & $-\phi^{\prime}(0)$ \\
\hline 0.0 & 0.1 & 2.0 & 1.0 & 1.0 & 0.952211 & 0.888412 \\
0.3 & & & & & 1.095247 & 1.012367 \\
0.6 & & & & & 1.255417 & 1.150977 \\
0.9 & & & & & 1.430970 & 1.302867 \\
0.5 & 0.0 & & & & 1.211527 & 1.114297 \\
& 0.2 & & & & 1.188867 & 1.092170 \\
& 0.4 & & & & 1.166147 & 1.070027 \\
& 0.6 & & & & 1.143697 & 1.048417 \\
& 0.1 & 0.0 & & & 1.284967 & 1.186447 \\
& & 1.0 & & & 1.200227 & 1.155257 \\
& 2.0 & & & 1.177467 & 1.080947 \\
& 2.5 & & & 1.050047 & 0.954721 \\
& & & & & & \\
& & 0.0 & & 1.139877 & 1.043827 \\
& & & 0.8 & & 1.178137 & 1.081527 \\
& & & 1.2 & & 1.220370 & 1.122887 \\
& & & & -0.2 & 1.174967 & 1.078047 \\
& & & & 0.5 & 1.182967 & 1.085977 \\
& & & & 1.0 & 1.253287 & 1.126987 \\
\hline
\end{tabular}

Table 3. Values of the local Nusselt number $R e_{x}^{-1 / 2} N u_{x}$ and the local Sherwood number $R e_{x}^{-1 / 2} S h$ for some values of $P r$, $D f, L e$, and $S r$ when $S=0.5, \beta=0.1, \lambda=N=1.0, \gamma=2.0$.

\begin{tabular}{cccccc}
\hline$P r$ & $D f$ & $L e$ & $S r$ & $-\theta^{\prime}(0)$ & $-\phi^{\prime}(0)$ \\
\hline 0.7 & 0.1 & 1.0 & 0.2 & 0.964282 & 0.866466 \\
1.2 & & & & 1.341770 & 1.253047 \\
1.5 & & & & 1.535157 & 1.469747 \\
2.0 & & & & 1.816057 & 1.816027 \\
1.0 & 0.0 & & & 1.277147 & 1.085967 \\
& 0.2 & & & 1.120797 & 1.120797 \\
& 0.4 & & & 0.953751 & 1.157167 \\
& 0.6 & & & 0.774757 & 1.195337 \\
& 0.1 & 0.7 & & 1.235027 & 0.859517 \\
& & 0.9 & & 1.211117 & 1.024237 \\
& & 1.2 & & 1.180170 & 1.255867 \\
& & 1.5 & & 1.152947 & 1.474567 \\
& & 1.0 & 0.0 & 1.122697 & 1.706747 \\
& & & 0.3 & 1.168227 & 1.354157 \\
& & & 0.5 & 1.199217 & 1.103987 \\
& & & 0.7 & 1.230967 & 0.840387 \\
\hline
\end{tabular}

values of $\hbar_{f}, \hbar_{\theta}$, and $\hbar_{\phi}$ are $-1.1 \leq \hbar_{f}, \hbar_{\theta}, \hbar_{\phi} \leq-0.2$. Our computation further show that the series given by (51) - (53) converge in the whole region of $\eta$ when $\hbar_{f}=\hbar_{\theta}=\hbar_{\phi}=-0.7$.

\section{Results and Discussion}

In order to see the influence of key parameters on $f^{\prime}, \theta$, and $\phi$, we plotted Figures $2-30$. The values of local Nusselt number $R e_{x}^{-1 / 2} N u_{x}$ and the local Sherwood number $R e_{x}^{-1 / 2} S h$ for various values of embedded parameters are also given in Tables 2 and 3 .

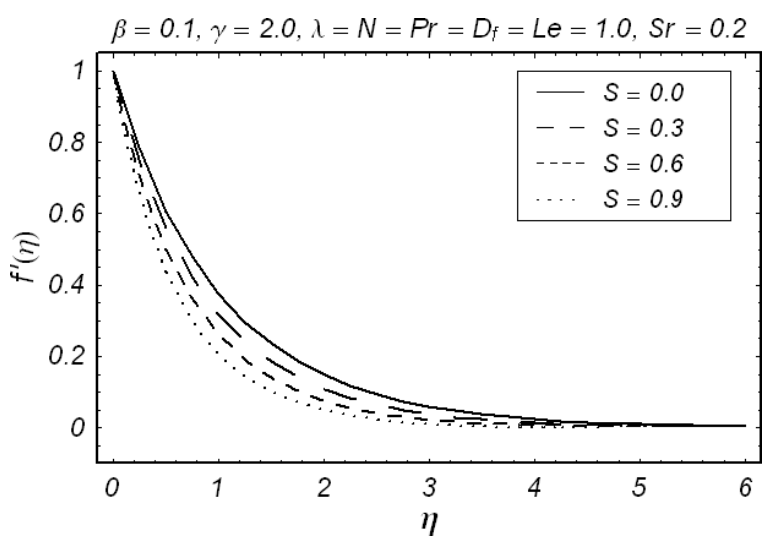

Fig. 2. Influence of $S$ on $f^{\prime}(\eta)$.

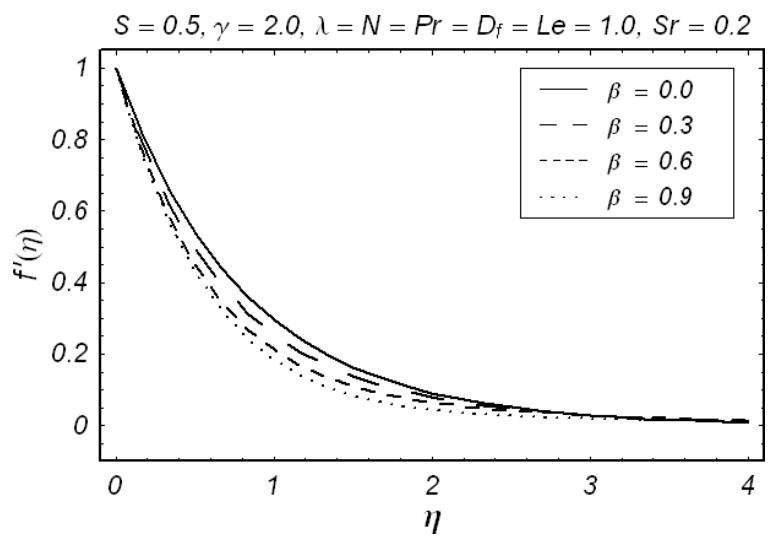

Fig. 3. Influence of $\beta$ on $f^{\prime}(\eta)$.

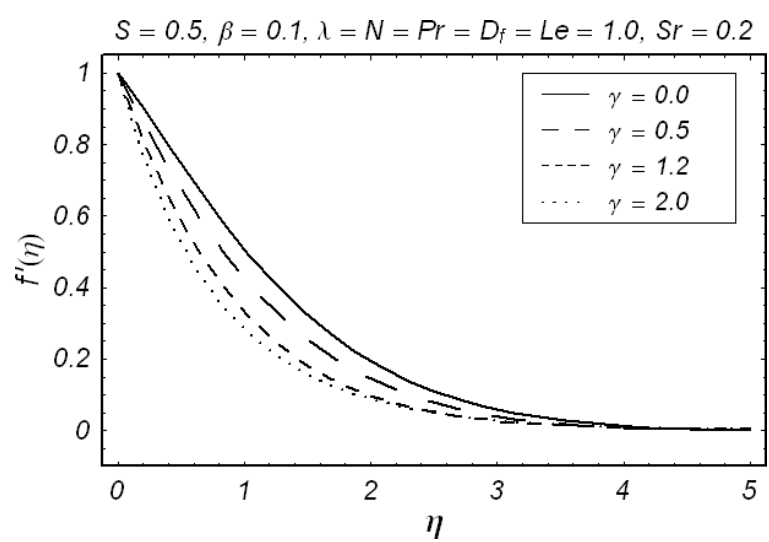

Fig. 4. Influence of $\gamma$ on $f^{\prime}(\eta)$.

Figures 2-11 show the effects of suction parameter $S$, the Deborah number $\beta$, the constant dimensionless porosity parameter $\gamma$, the constant dimensionless mixed convection parameter $\lambda$, the concentration buoyancy parameter $N$, the Prandtl number $\operatorname{Pr}$, the 


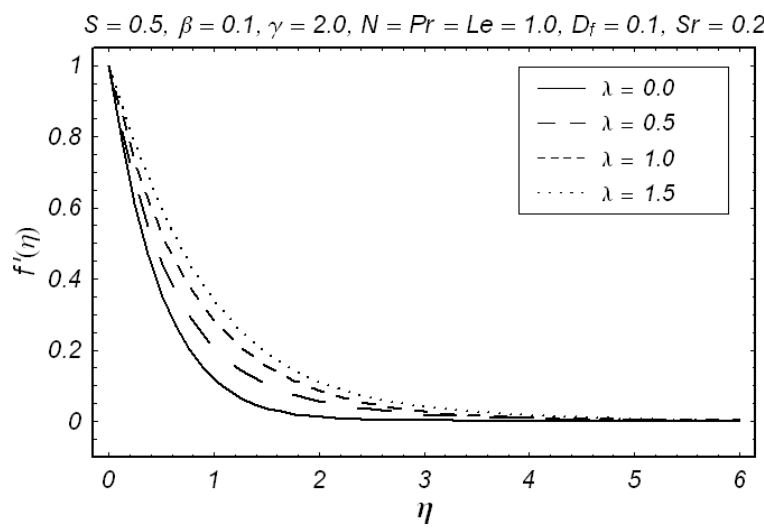

Fig. 5. Influence of $\lambda$ on $f^{\prime}(\eta)$.

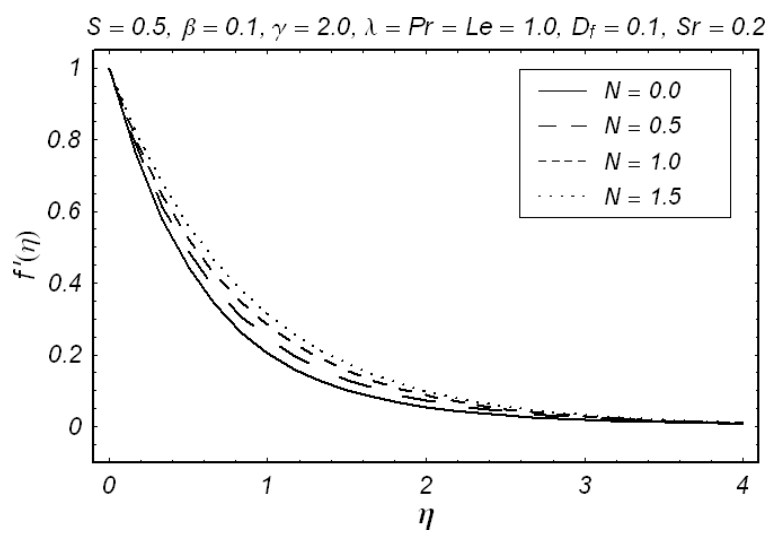

Fig. 6. Influence of $N(>0)$ on $f^{\prime}(\eta)$.

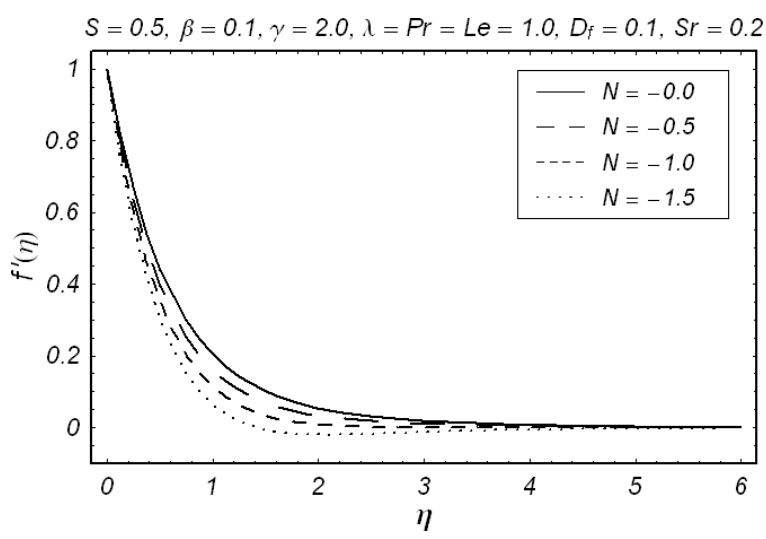

Fig. 7. Influence of $N(<0)$ on $f^{\prime}(\eta)$.

Lewis number $L e$, the Dufour number $D f$, and the Soret number $S r$ on $f^{\prime}$. The velocity field and boundary layer thickness increase by increasing $\lambda, D f$, and $S r$. On the other hand the velocity field and the boundary layer thickness decrease when $S, \beta, \gamma, P r$, and $L e$

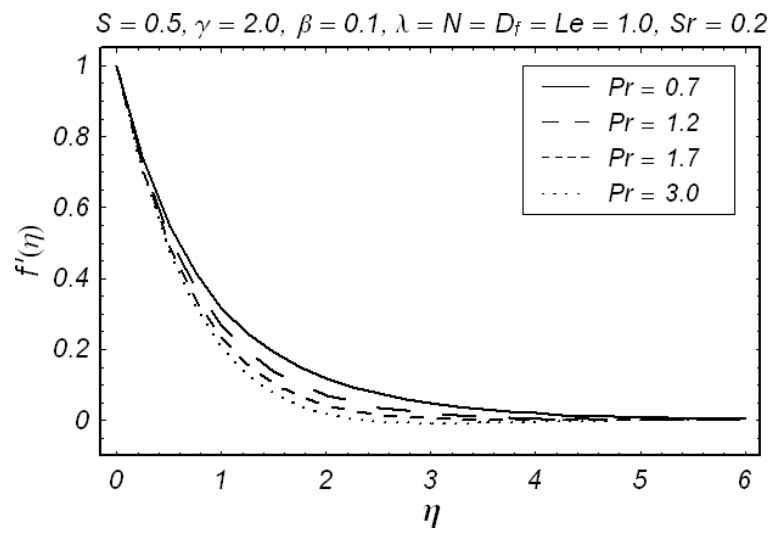

Fig. 8. Influence of $\operatorname{Pr}$ on $f^{\prime}(\eta)$.

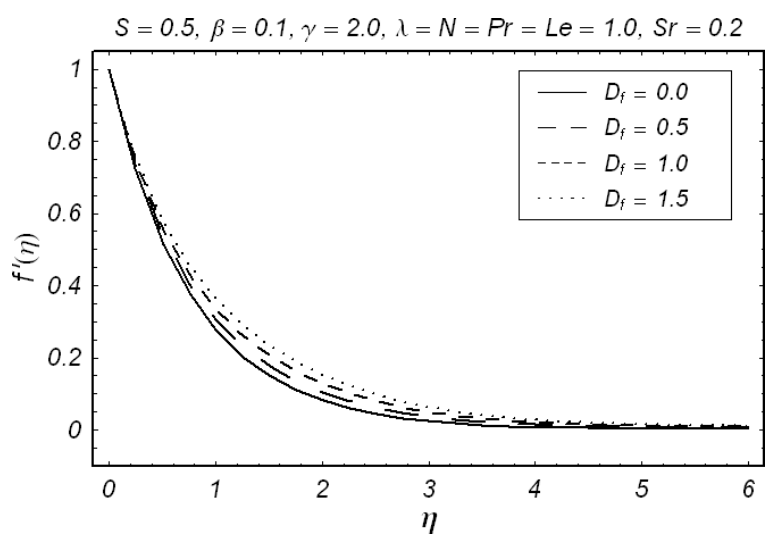

Fig. 9. Influence of $D f$ on $f^{\prime}(\eta)$.

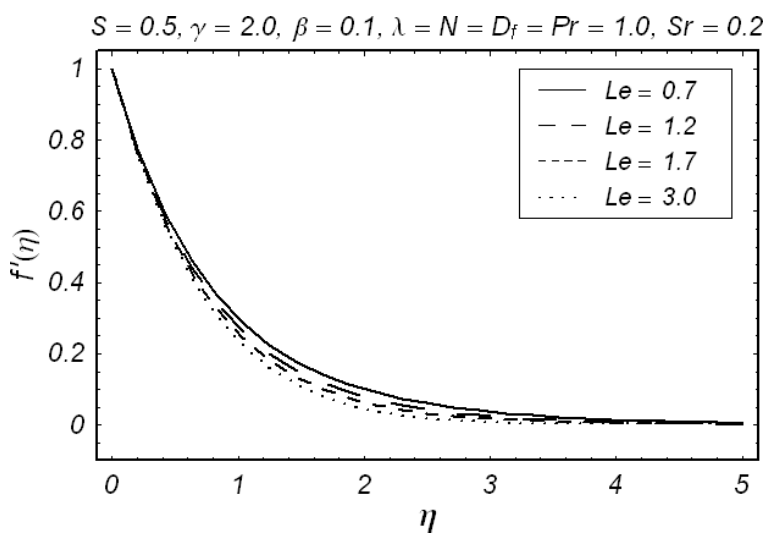

Fig. 10. Influence of Le on $f^{\prime}(\eta)$.

are increased. The influence of $N$ for both the cases is analysed in Figures 6 and 7. These figures show that the velocity and the boundary layer thickness increase for positive values of $N$, while it decreases for its negetive values. Figures $12-20$ plot the behaviours 


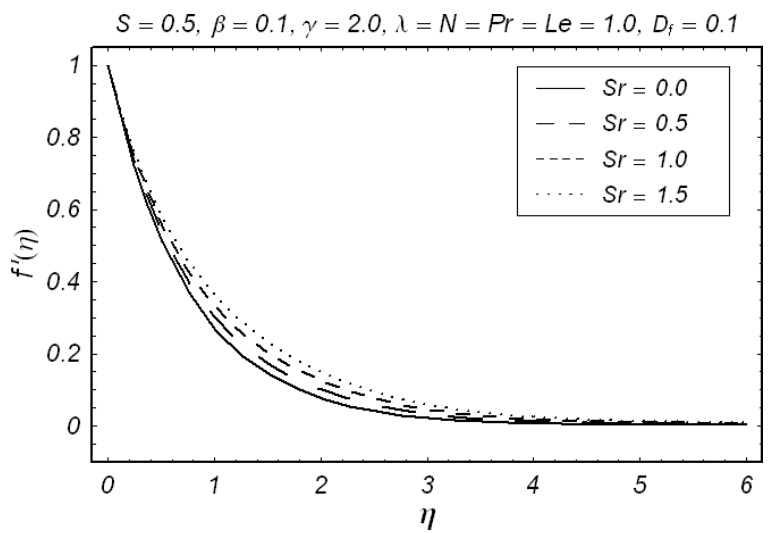

Fig. 11. Influence of $\operatorname{Sr}$ on $f^{\prime}(\eta)$.

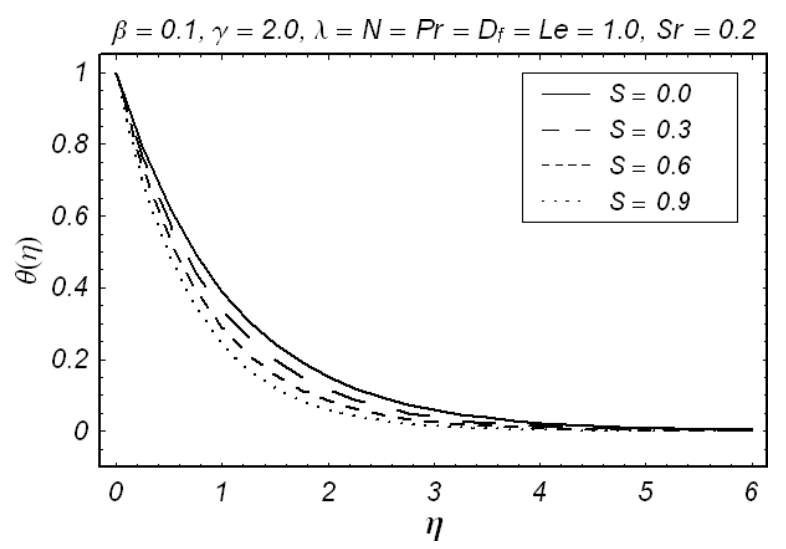

Fig. 12. Influence of $S$ on $\theta(\eta)$.

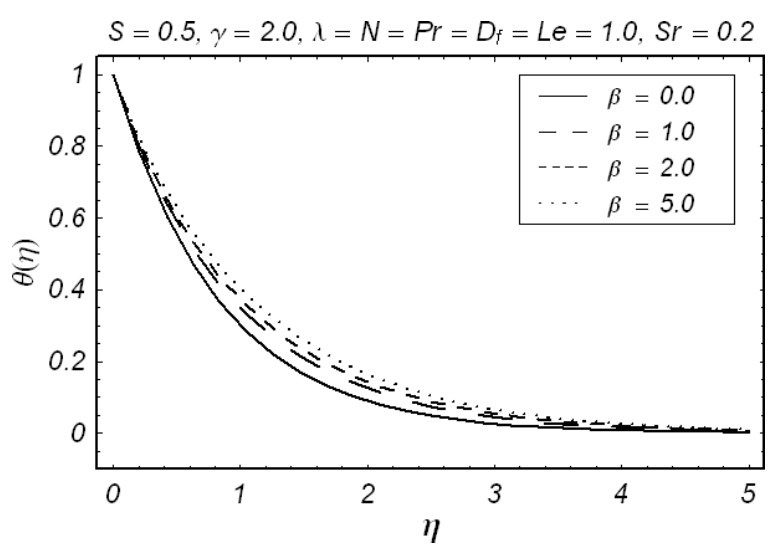

Fig. 13. Influence of $\beta$ on $\theta(\eta)$.

of $S, \beta, \gamma, \lambda, N, \operatorname{Pr}, D f$, and $S r$ on the temperature $\theta$. These figures depict that the temperature and the thermal boundary layer thickness increase upon increasing $\beta, \gamma$, and $S r$, whereas temperature and the thermal boundary layer thickness decrease when $S, \lambda, \operatorname{Pr}, D f$,

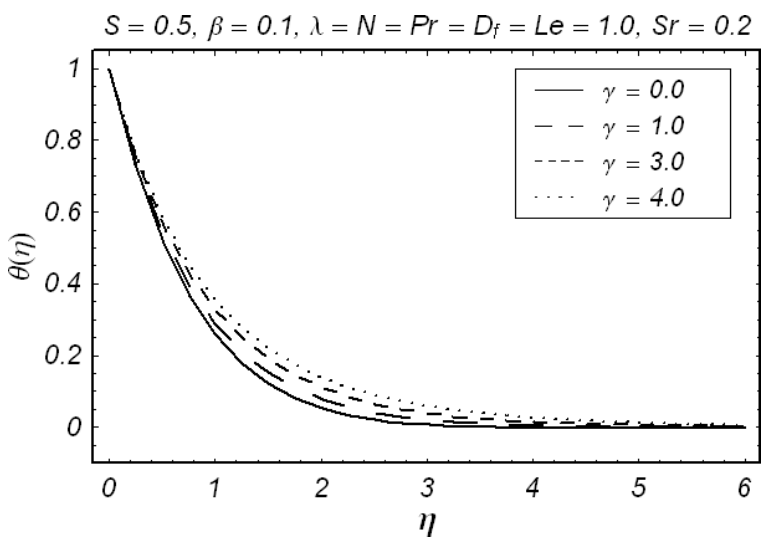

Fig. 14. Influence of $\gamma$ on $\theta(\eta)$.

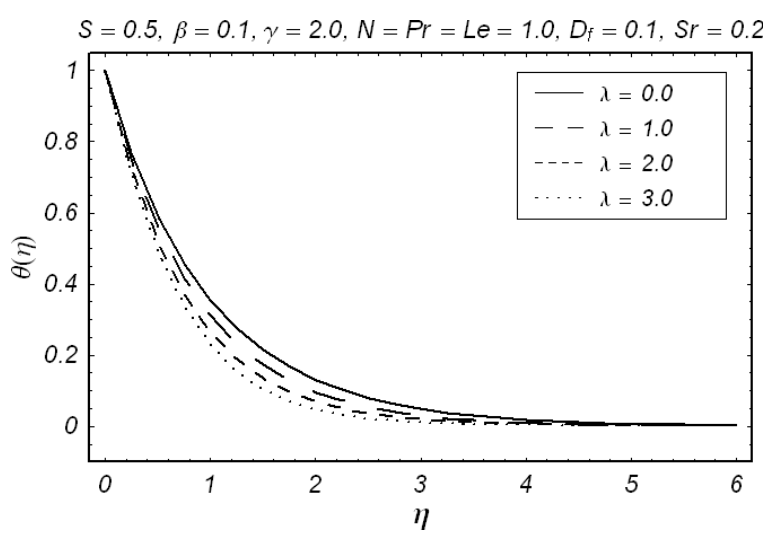

Fig. 15. Influence of $\lambda$ on $\theta(\eta)$.

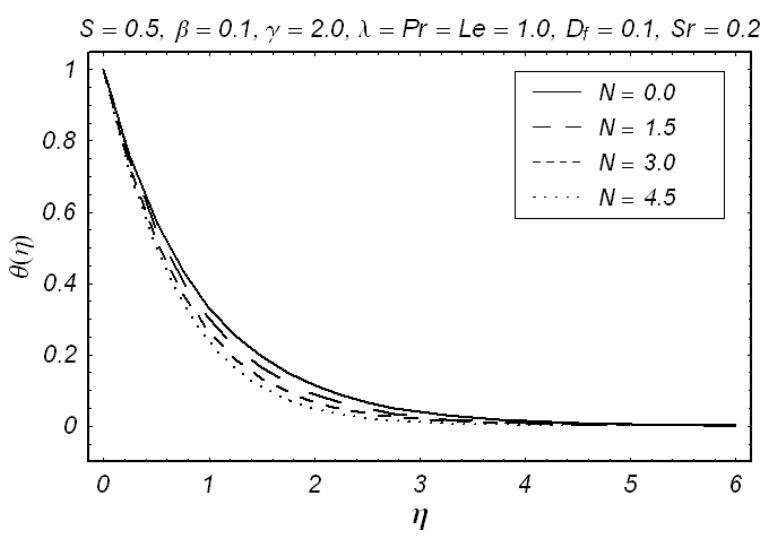

Fig. 16. Influence of $N(>0)$ on $\theta(\eta)$.

and $L e$ are increased. The effects of $N$ are displayed in Figures 16 and 17. The temperature and thermal boundary layer thickness decrease for positive values of $N$. However, it decreases for negative values of $N$. Figures 21-30 plot the behaviour of these parameters 


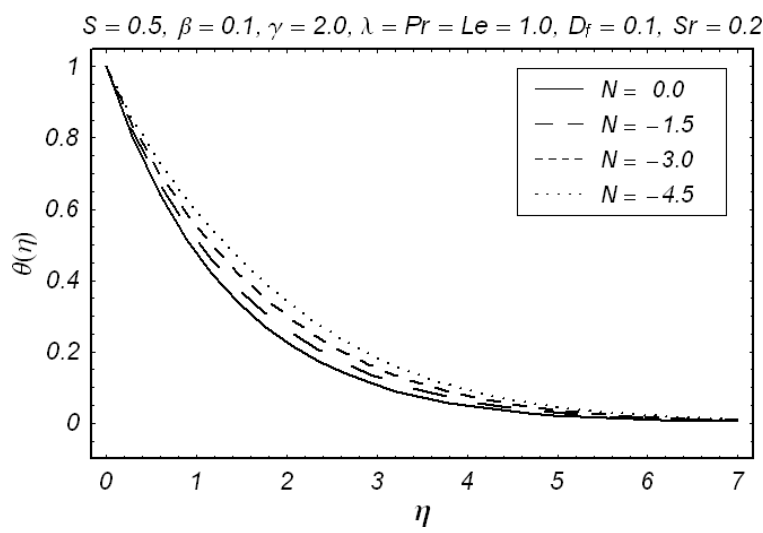

Fig. 17. Influence of $N(<0)$ on $\theta(\eta)$.

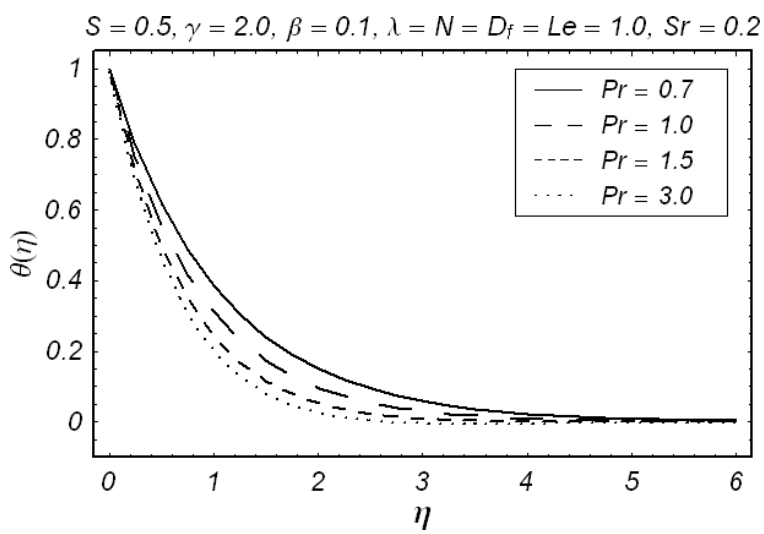

Fig. 18. Influence of $\operatorname{Pr}$ on $\theta(\eta)$.

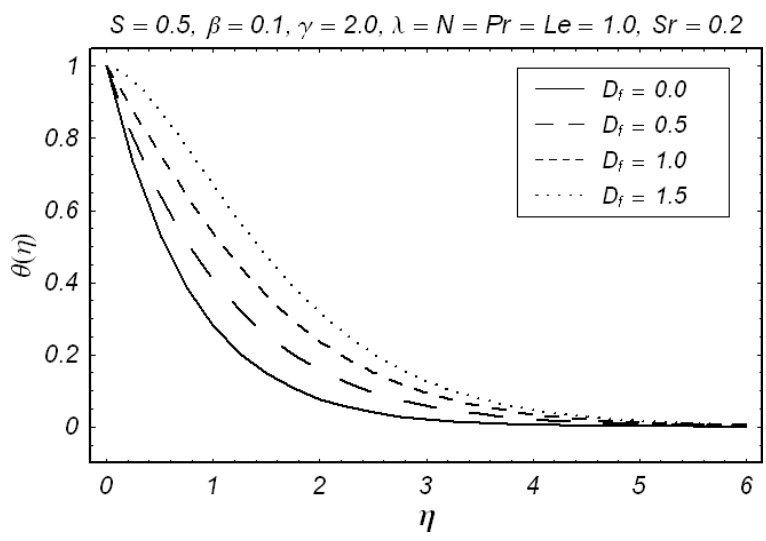

Fig. 19. Influence of $D f$ on $\theta(\eta)$.

on the concentration field $\phi$. These figures show that $\phi$ increases upon increasing $\beta, \gamma$, and $S r$, whereas $\phi$ decreases when $S, \lambda, \operatorname{Pr}, D f$, and $L e$ are increased. The effects of $N$ on the concentration field are similar to those of temperature.

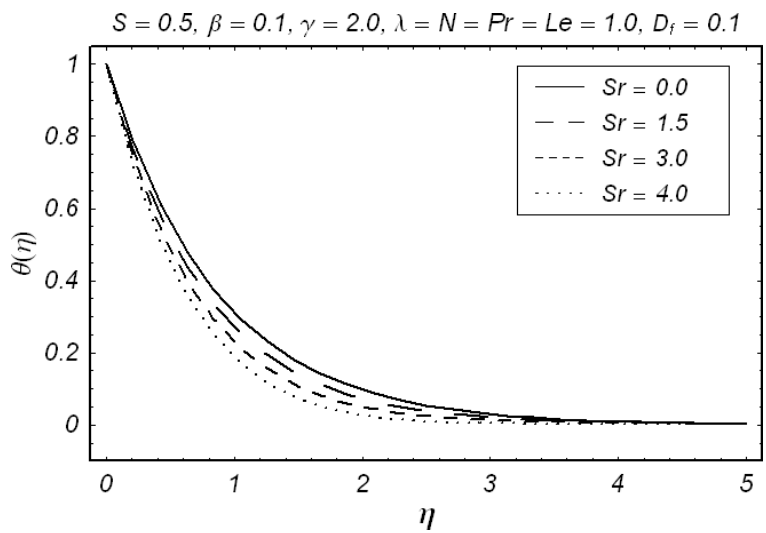

Fig. 20. Influence of $S r$ on $\theta(\eta)$.

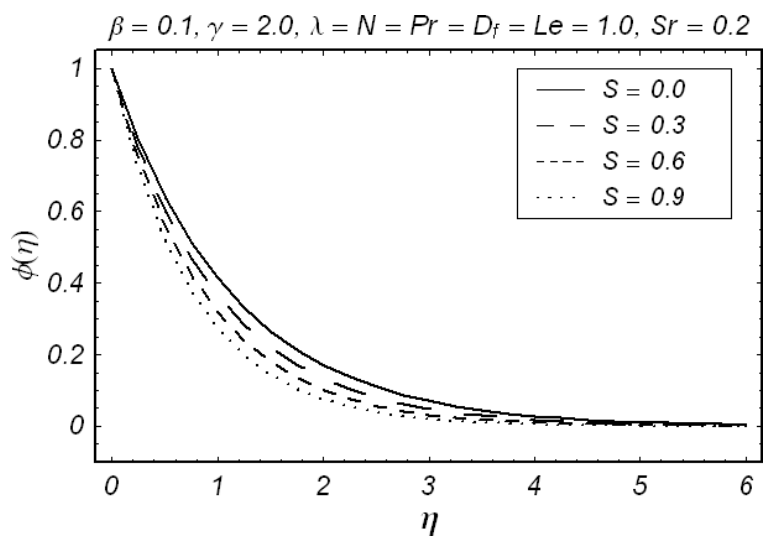

Fig. 21. Influence of $S$ on $\phi(\eta)$.

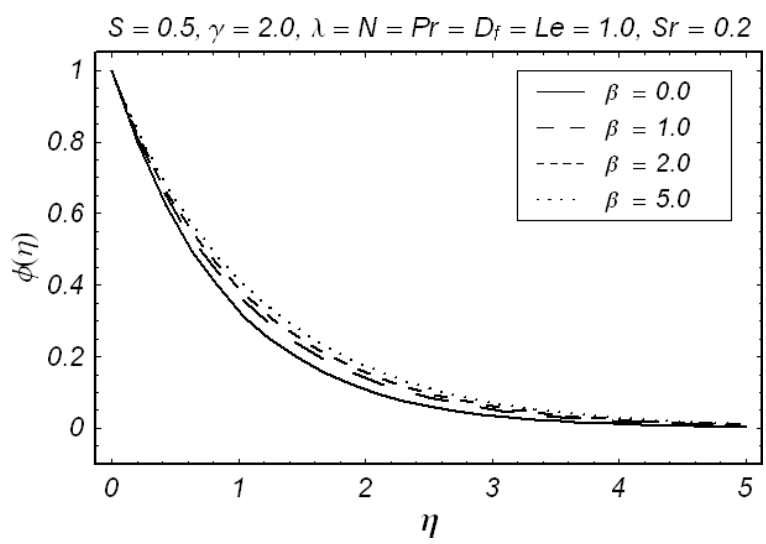

Fig. 22. Influence of $\beta$ on $\phi(\eta)$.

Table 1 is prepared for the convergence of the series solutions. Obviously, the convergence is achieved at 15 th-order of approximations. Tables 2 and 3 show the values of the local Nusselt number $R e_{x}^{-1 / 2} N u_{x}$ and the local Sherwood number $R e_{x}^{-1 / 2} S h$. The magnitude 

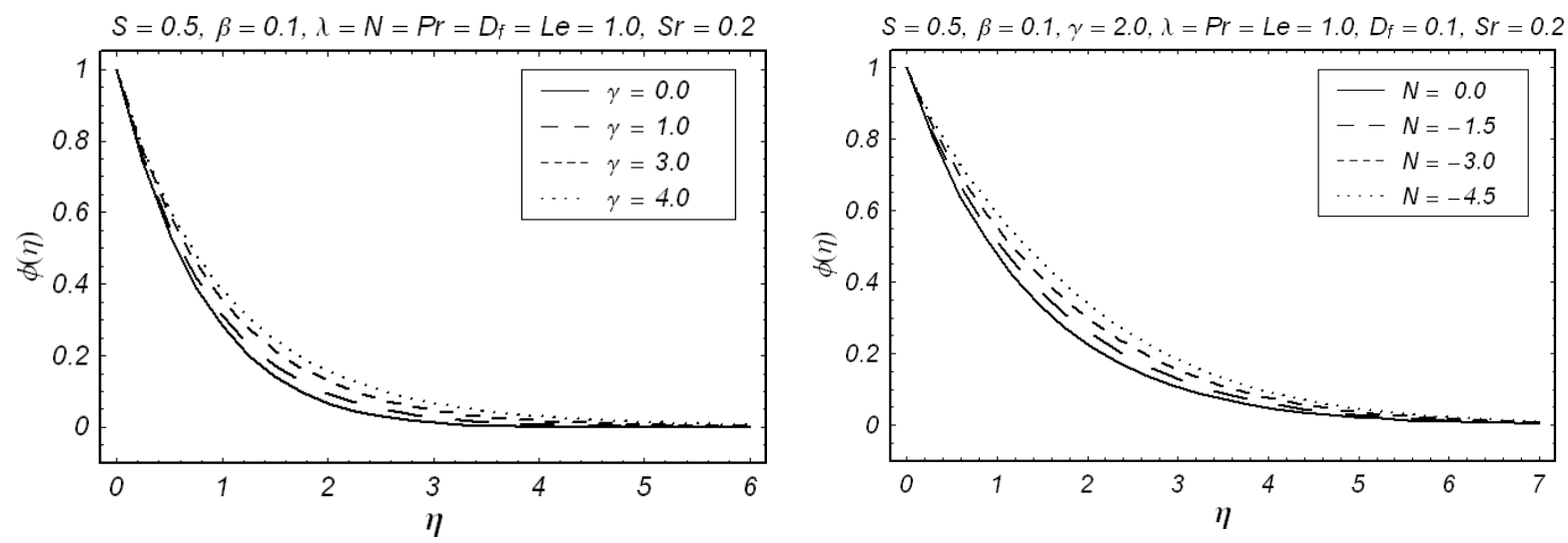

Fig. 23. Influence of $\gamma$ on $\phi(\eta)$.

Fig. 26. Influence of $N(<0)$ on $\phi(\eta)$.
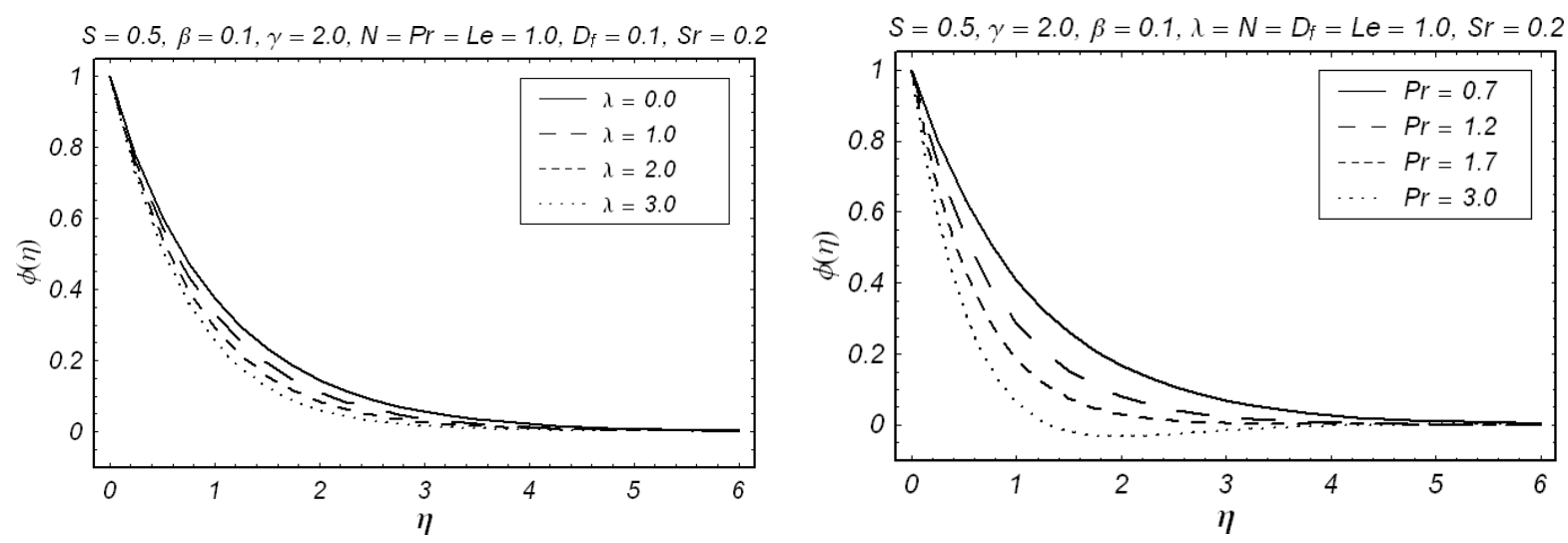

Fig. 24. Influence of $\lambda$ on $\phi(\eta)$.

Fig. 27. Influence of $\operatorname{Pr}$ on $\phi(\eta)$.
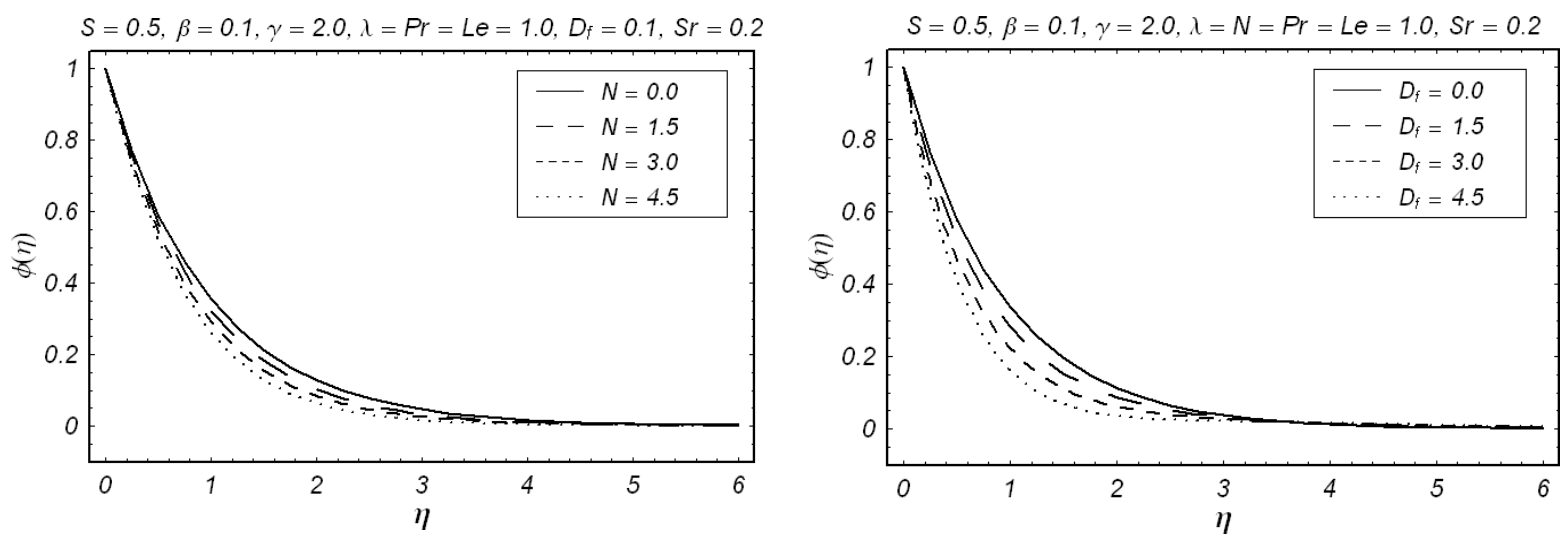

Fig. 25. Influence of $N(>0)$ on $\phi(\eta)$.

of the local Nusselt number increases for large values of $S, \lambda, P r$, and $S r$. However it decreases for large values of $\beta, \gamma, D f$, and $L e$. The magnitude of the local

Fig. 28. Influence of $D f$ on $\phi(\eta)$.

Sherwood number increases for large values of $S, \lambda$, $\operatorname{Pr}, D f$, and $L e$, whereas it decreases for large values of $\beta, \gamma$, and $S r$. 


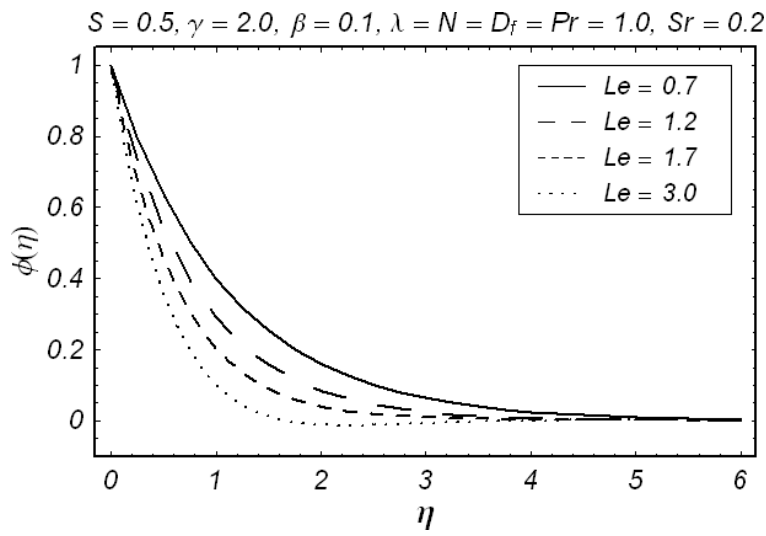

Fig. 29. Influence of $L e$ on $\phi(\eta)$.

[1] C. Fetecau and C. Fetecau, Int. J. Eng. Sci. 43, 781 (2005).

[2] C. Fetecau, M. Athar, and C. Fetecau, Comput. Math. Appl. 57, 596 (2009).

[3] C. Fetecau and C. Fetecau, Int. J. Eng. Sci. 44, 788 (2006).

[4] W. C. Tan, P. W.-Xiao, and X. M. Yu, Int. J. Nonlinear Mech. 38, 645 (2003).

[5] K. S. Mekheimer and Y. Abd Elmaboud, Physica A 387, 2403 (2008).

[6] M. H. Haroun, Commun. Nonlinear Sci. Numer. Simul. 12, 1464 (2007).

[7] T. Hayat, Z. Abbas, and M. Sajid, Phys. Lett. A 372, 2400 (2008).

[8] Z. Abbas, Y. Wang, T. Hayat, and M. Oberlack, Int. J. Nonlinear Mech. 43, 783 (2008).

[9] T. Hayat, M. Sajid, and M. Ayub, Commun. Nonlinear Sci. Numer. Simul. 13, 745 (2008).

[10] T. Hayat, M. A. Farooq, T. Javed, and M. Sajid, Nonlinear Anal.: Real World Appl. 10, 745 (2009).

[11] S. J. Liao, Commun. Nonlinear Sci. Numer. Simul. 14, 2144 (2009).

[12] H. Xu, S. J. Liao, and X. C. You, Commun. Nonlinear Sci. Numer. Simul. 14, 1152 (2009).

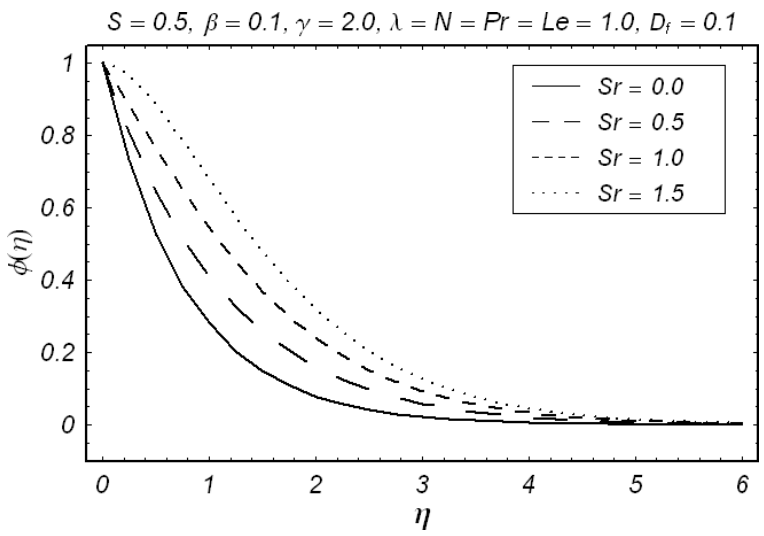

Fig. 30. Influence of $S r$ on $\phi(\eta)$.

[13] H. Xu and S. J. Liao, Commun. Nonlinear Sci. Numer. Simul. 13, 350 (2008).

[14] S. J. Liao, Commun. Nonlinear Sci. Numer. Simul. 14, 983 (2009).

[15] Y. Tan and S. Abbasbandy, Commun. Nonlinear Sci. Numer. Simul. 13, 539 (2008).

[16] S. Abbasbandy, Phys. Lett. A 372, 613 (2008).

[17] S. Abbasbandy and F. S. Zakaria, Nonlinear Dyn. 51, 83 (2008).

[18] S. Kechil and I. Hashim, Commun. Nonlinear Sci. Numer. Simul. 14, 1346 (2009).

[19] I. Hashim, O. Abdulaziz, and S. Momani, Commun. Nonlinear Sci. Numer. Simul. 14, 674 (2009).

[20] M. Sajid and T. Hayat, Int. Commun. Heat Mass Transfer 35, 3457 (2008).

[21] M. Sajid, T. Hayat, and S. Asghar, Int. J. Heat Mass Transfer 50, 1723 (2007).

[22] M. Sajid and T. Hayat, Choas, Soliton, and Fractals 38 , 506 (2008).

[23] M. Sajid, I. Ahmed, T. Hayat, and M. Ayub, Commun. Nonlinear Sci. Numer. Simul. 13, 2193 (2008).

[24] T. Hayat, T. Javed, and M. Sajid, Phys. Lett. A 372, 3264 (2008).

[25] M. Sajid and T. Hayat, Int. Commun. Heat Mass Transfer 36, 59 (2009). 